\title{
Research on Bending Fatigue Properties of Reinforced Macadam Foundation
}

\author{
Hongjun JING*, Mingjie GOU, Lichen SONG
}

\begin{abstract}
Existing macadam base structures have poor resistance to bending deformation of pavement. The structural layer is challenged with serious cracking due to the long-term dynamic loads. Despite these issues, however, studies on reinforcement techniques for macadam base structure have been scarce. No reasonable and feasible method on how to improve the bending resistance of flexible base structures (e.g. macadam base structure) and prolong their bending fatigue life has yet been reported. Thus, this study proposed a method for reinforcing macadam base structures with geogrids to strengthen bending performance, anti-fatigue performance and dynamic stability of flexible base structures under different loading levels. The reinforced macadam base structure was investigated through a laboratory test of flexural-tensile strength and a three-point fatigue bending test. A cyclic loading test of reinforced and non-reinforced girder specimens was carried out under five stress levels. The effect of such a new reinforcement method in improving the bending fatigue properties of macadam base structures was analyzed by comparing the yield curves and fatigue lives of reinforced and non-reinforced specimens under different loading levels. Results demonstrate that the non-reinforced macadam base has poor bending resistance. The yield curve and fatigue life of non-reinforced specimens attenuated quickly as it approached the ultimate loading level. Non-reinforced specimens have low-cycle fatigue failures when the loads reach the 0.8 stress level and the ultimate displacement at failure is relatively small, accompanied by obvious failure surface. After geogrids are added, the fatigue life of specimens improved significantly. The yield curve shows no significant attenuation at a high loading level. The reinforced specimens can still maintain high-cycle fatigue failure under the 0.8 stress level and avoid the occurrence of low-cycle fatigue failures. Moreover, reinforced specimens can inhibit transmission of reflection cracks at midspan of cement-stabilized macadam base, delay the propagation of oblique shearing fractures close to the support, and strengthen the dynamic stability and durability of pavement structure with a macadam base. The conclusions of this study provide theoretical references to practical engineering applications of such new reinforcement technology for macadam bases.
\end{abstract}

Keywords: bending fatigue test; fatigue life; flexible base; geogrid; reinforced macadam foundation

\section{INTRODUCTION}

In recent years, geogrid reinforcement technology has been increasingly applied in subgrade engineering with the development of base structures for composite pavement. In engineering, geogrids are generally paved in the space between the semi-rigid base and the asphalt surface to improve reflection cracks on the surface. However, the pavement structure of the traditional semi-rigid base is restricted by the rigidity of materials and it cannot inhibit the initiation and transmission of reflection cracks effectively. Accordingly, geogrid reinforcement also cannot provide the best effect. Hence, a composite reinforced macadam base structure characteristic of simple structure and convenient construction was proposed to solve reflection cracks on pavement better and realize multiple functions and high durability of the base. This composite reinforced macadam foundation structure is crucial to the improvement of overall durability and dynamic stability of roads.

The traditional semi-rigid base has serious reflection cracks. Scholars have carried out studies on the reinforcement mode of the base [1-4]. Many scholars have suggested that geogrid reinforcement could strengthen the dynamic stability of pavements effectively. The influence of reinforcement material properties on the reinforcement effect [5-7] and the theoretical calculation model of reinforcement [8-11] have also been discussed. Some scholars have carried out laboratory full-size fatigue experimental studies on reflection cracks of pavement [1214]. However, experimental results have difficulty reflecting the general laws of reinforced composite base structures because of high experimental cost, few samples, and nonartificial errors in the experiment process. With the continuous development of flexible base structures, new high-efficiency anti-cracking structures, such as macadam anti-cracking buffer layers, have been developed. Although such anti-cracking structures have stronger cracking resistance than the traditional semi-rigid base structures, they are disadvantageous because of their relatively low rigidity, loose structure and no cementation. When the subgrade sinks and separates from the sub-base, bending failures under fatigue dynamic loads can easily develop in the base structure. At present, few studies on bending fatigue properties of macadam base structures have been conducted. No feasible method to improve bending fatigue properties of macadam base structures has also been proposed, which restricts the development of composite macadam anticracking structures.

This study proposes strengthening the macadam base with geogrids and improves bending fatigue properties of composite macadam base structures to eliminate the disadvantages highlighted in previous studies. For this purpose, summarizing and analyzing existing studies are necessary. Considering the disadvantages of full-size fatigue tests, a three-point bending fatigue laboratory test was proposed to investigate bending failure laws and bending fatigue properties of composite reinforced macadam base structures.

\section{STATE OF THE ART}

The macadam flexible base is a new anti-cracking structure that has emerged recently. It has a loose structure without cementation and offsets vehicle loads on the structural layer through the rearrangement of particles. Many studies on macadam flexible bases have been conducted. Li et al. [15] constructed a refined model based on ABAQUS software to explore the mechanical responses of the flexible base of airports above its underground infrastructure under wheel loads and found that the wheel loads at the aligned positions with sides of the pipeline section are the most critical loading conditions. Valli et al. [16] discussed the effects of natural rubber, carbon black and copper slag in flexible base structures and determined that adding natural rubber in flexible materials could 
strengthen vibration absorption performances of the structural layer, adding copper slag could improve the mechanical properties of the mixture, and adding carbon black could improve the tear strength, conductivity and elasticity of asphalt. Öcal et al. [17] proposed an antiinference method that combined the neural network computation method to estimate the material properties of flexible asphalt pavement and brought finite element software simulation results and field test data into the analysis. Results demonstrated that the proposed antiinference method could determine the properties of materials accurately and quickly. Based on the dynamic responses of multi-layer anisotropic flexible pavement with linear gradient interlayers to simple harmonic vibration loads, You et al. [18] developed a new analytic method using Fourier waveform combination variation. The Fourier waveform combination variation method has been proven to be applicable in the design of multi-layer flexible pavement structure based on computer programassisted verification. To improve the quality of regur soils, which are common in India, Patil et al. [19] added a powder inorganic admixture (RBI grade 81) into the regur soils based on certain proportions and formed a flexible base structure after $7 \mathrm{~d}$ of curing. The dosages of different materials were also designed. Following existing standards in India, Sayyed et al. [20] carried out a finite element analysis on the optimum section of flexible pavement in terms of bearing capacity by combining them with the practical design method and proved the validity of the finite element analysis. Results showed that excessively soft asphalt mixture might decrease the structural performances of pavement under high temperature, while excessively hard asphalt mixture may cause the pavement to be brittle under low temperature, thereby resulting in surface cracking under loads. Habbouche et al. [21] suggested that the experimental stress relief course (ESRC) buffer layer can be used as an effective method to relieve reflection cracks on asphalt coating. The effect of the ESRC buffer layer in inhibiting reflection cracks on asphalt coating was proved through a field comparative test. Peng et al. [22] discussed the effects of asphalt-poor macadam buffer layer in relieving reflection cracks on the semi-rigid foundation by simulating practical wheel loads. They found that such asphalt-poor macadam had good pavement performance and that it can be used as an anti-reflection buffer layer for the semi-rigid base. Pan et al. [23] suggested relieving reflection cracks in pavements by using warm-mix asphalt modified mixture as the stress absorption layer. Its good anti-cracking effect was verified through a finite element analysis and a laboratory test.

Previous literature shows that many scholars have carried out numerical simulation analyses and laboratory experimental studies on material properties and anticracking effects of flexible macadam stress absorption layer. Although these studies found that a flexible base can improve reflection cracks on the surface, few studies have focused on the bending fatigue properties of flexible macadam base structures. Many studies have demonstrated that the flexible anti-cracking buffer layer cannot control bending fatigue cracks effectively and prolong the service life of base structures for pavement under various loading conditions. Moreover, evaluation and studies on bending fatigue properties of flexible anti-cracking buffer layers are also lacking.

To strengthen anti-cracking performances of foundation structures, some scholars have suggested reinforcing the base with geogrids and discussed the reinforcement effect through laboratory tests. GonzalezTorre et al. [24] carried out a comparative study on the anticracking effect of six types of geosynthetics through a laboratory test. They found that geogrid was superior to geotextiles in terms of anti-reflection cracks and the best effect of anti-reflection cracks can be achieved by using geogrids with a high secant modulus value. Saride et al. [25] discussed the effects of geogrids in inhibiting reflection cracks and improving fatigue performances of the asphalt overlay through a bending fatigue test and digital image correlation (DIC) technology. Results demonstrated that geogrids with high interface shearing and tensile bonding performance can effectively inhibit reflection cracks and improve the fatigue life of the overlay. Correia et al. [26] studied the mechanical properties of geogrid-reinforced flexible pavement through a self-made cyclic wheel loading device for pavement test and found that geogrids can not only strengthen the structural performances of flexible pavement but also decrease tracks and permanent lateral movement of the surface layer. Hadi et al. [27] analyzed the reinforcement effect of geogrids on fatigue performance of concrete pavement and proposed an equation of concrete pavement under cyclic loads. Saha et al. [28] carried out a series of laboratory model tests on non-reinforced and reinforced wet macadam mixtures to discuss settlement characteristics and determined that the bearing capacity of reinforced wet macadam mixture was significantly higher. Sun et al. [29] deduced a solution to the typical stabilized flexible pavement with three geosynthetics layers based on the hierarchical elastic theory. They also applied the deduced solution to the empirical method of calculating track and fatigue cracking and achieved good performance.

In the above studies, the effects of geogrids in improving reflection cracks of base based on laboratory tests were discussed. These studies concluded that geogrid reinforcement can decrease the number of cracks on the semi-rigid asphalt pavement surface. However, single geogrid reinforcement can only control open fractures caused by traverse tensile forces but not the vertical shearing cracks caused by uncoordinated deformations between cement-stabilized macadam base and asphalt surface effectively. Therefore, achieving a pavement with true long service life through geogrid reinforcement alone is difficult.

To address existing research disadvantages, this study suggested reinforcing the flexible macadam base with geogrids to improve the bending fatigue properties of the base structure. The geogrid-reinforced macadam base and cement-stabilized macadam base were applied to form a composite base structure for the comprehensive control of various reflection cracks on the pavement. A laboratory bending strength test and three-point bending fatigue test were performed on reinforced and non-reinforced macadam base structures. Bending resistance and fatigue life of reinforced macadam base structures and improvement effect of geogrid reinforcement on antireflection crack performances were analyzed using the test 
results of reinforced and non-reinforced specimens under different loading levels. Test results proved that reinforced macadam base structures have good bending resistance and its dynamic stability under high loading levels can be strengthened effectively by geogrids.

\section{METHODOLOGY}

3.1 Test Conditions

\subsubsection{Specimen Design and Manufacturing}

In this study, a comparative test between reinforced and non-reinforced specimens was carried out. The sizes of the reinforced and non-reinforced specimens were both $150 \times 150 \times 550 \mathrm{~mm}$. Geogrids were paved in the space between the cement-stabilized macadam base and the grading macadam base. The thickness of all structures was determined to be $50 \mathrm{~mm}$. Aggregate grading of different structures of specimens is shown in Tab. 1, Tab. 2 and Tab. 3.

Table 1 Grading scope of asphalt-concrete aggregate

\begin{tabular}{|c|c|c|c|c|c|c|c|c|c|c|c|c|c|c|}
\hline Screen size / mm & & 16 & 13.2 & 9.5 & \multicolumn{2}{|c|}{4.75} & \multicolumn{2}{|c|}{2.36} & 1.18 & 0.6 & 0.3 & \multicolumn{2}{|r|}{0.15} & 0.075 \\
\hline Through rate / \% & & 100 & $90-100$ & $68-85$ & $38-$ & & & -50 & $15-38$ & $10-28$ & $7-20$ & & $5-15$ & $4-8$ \\
\hline Screen size $/ \mathrm{mm}$ & 315 & 26.5 & 19 & 16 & 13.2 & & & 4.75 & 2.36 & 118 & 0.6 & 03 & 0.15 & 0.075 \\
\hline Through rate / \% & 100 & $90-100$ & $71-85$ & $68-75$ & $54-69$ & & 54 & $30-40$ & $17-27$ & $12-18$ & $8-14$ & $6-8$ & $4-7$ & $2-5$ \\
\hline
\end{tabular}

Table 3 Grading scope of cement-stabilized macadam aggregate

\begin{tabular}{|c|c|c|c|c|c|c|c|c|c|c|}
\hline Screen size / mm & 37.5 & 31.5 & 26.5 & 19 & 9.5 & 4.75 & 2.36 & 0.6 & 0.075 \\
\hline Lower limit & 100 & $90-100$ & - & $67-90$ & $45-68$ & $29-50$ & $18-38$ & $8-22$ & $0-7$ \\
\hline
\end{tabular}

\begin{tabular}{|c|c|c|}
\multicolumn{2}{|c}{ Table 4 Compaction test results } \\
\hline Type of aggregates & $\begin{array}{c}\text { Maximum dry density / } \\
\mathrm{g} / \mathrm{cm}^{3}\end{array}$ & $\begin{array}{c}\text { Optimum moisture } \\
\text { content / \% }\end{array}$ \\
\hline Graded macadam & 2.306 & 4.9 \\
\hline $\begin{array}{c}5 \% \text { cement-stabilized } \\
\text { macadam }\end{array}$ & 2.280 & 5.5 \\
\hline
\end{tabular}

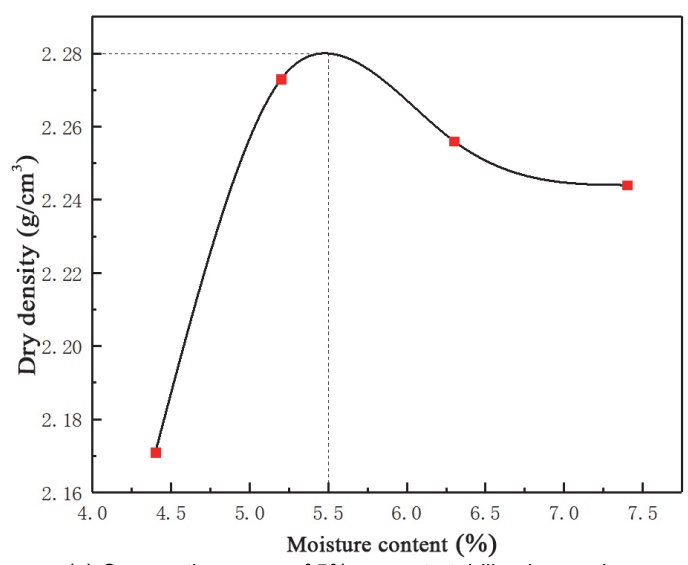

(a) Compaction curve of $5 \%$ cement-stabilized macadam

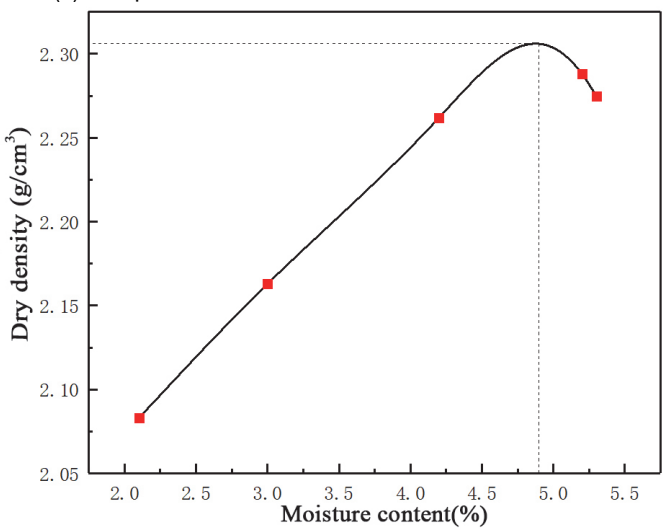

(b) Compaction curve of graded macadam

Figure 1 Compaction curves

A compaction test was performed on cement-stabilized macadam and graded macadam aggregates that meet the above grading scopes before preparation of specimens to determine the laboratory maximum dry density and moisture content. The results are shown in Tab. 4. The compaction curves of these aggregates are shown in Fig. 1.

Specimens were manufactured by a self-made vibrating compression table to ensure uniform distribution of aggregates. During compression, a piece of rigid pressing block was placed over the aggregate, and the pressure was applied with two large-tonnage jacks. A stable output of pressure during loading was ensured. Details are shown in Fig. 2.

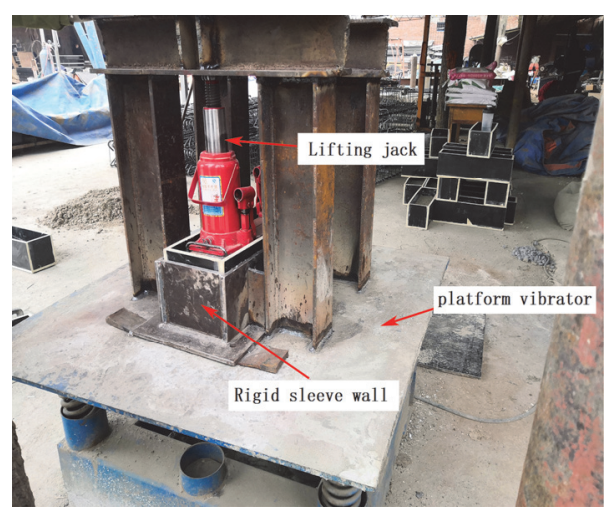

(a) Vibrating compression table under service

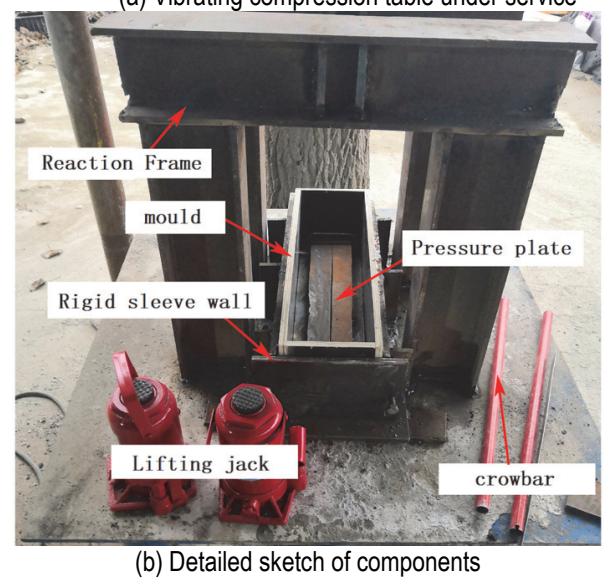

Figure 2 Self-made vibrating compression table 
The wooden mold was applied to manufacture the specimens. A total of 60 girder specimens were made and divided equally into Groups A and B according to the use of geogrids. Each group was further divided into five stress levels with each level having 5 specimens. All prepared specimens were cured in a standard chamber (temperature $=20{ }^{\circ} \mathrm{C} \pm 2{ }^{\circ} \mathrm{C}$ and humidity $\geq 95 \%$ ) for 90 days. Detailed parameters of specimens are listed in Fig. 3.

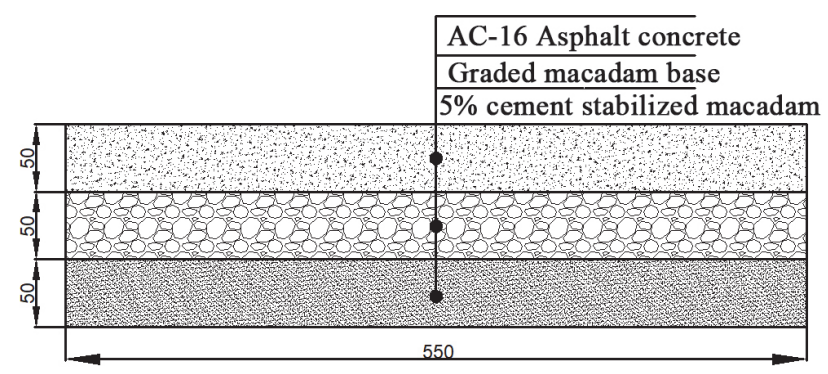

(a) Group A

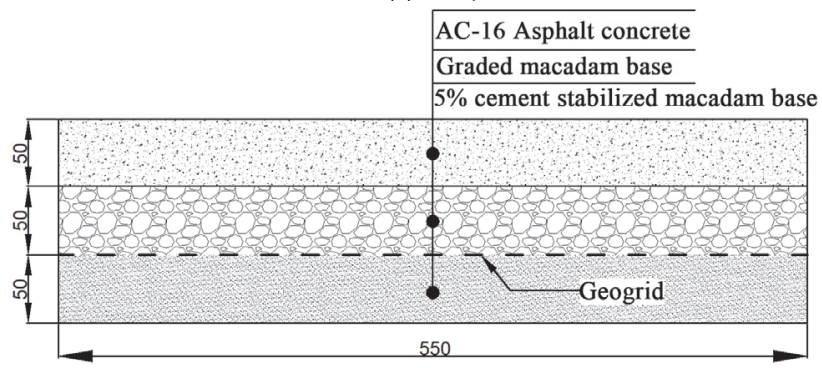

(b) Group B

Figure 3 Parameters of specimens

\subsubsection{Test Devices and Loading Schemes}

In this experiment, bending loads were applied onto all girder specimens in three points by using the MTS810 servo fatigue loader (Fig. 4). During laboratory loading, the distance of the spherical movable support was first set to $300 \mathrm{~mm}$. Then, the specimens were placed on the spherical movable support and three points were marked. The loading direction was kept constant with the pressure direction during the compaction of specimens. The upward and downward pressure heads were at three points of the specimens. Details are shown in Fig. 5.

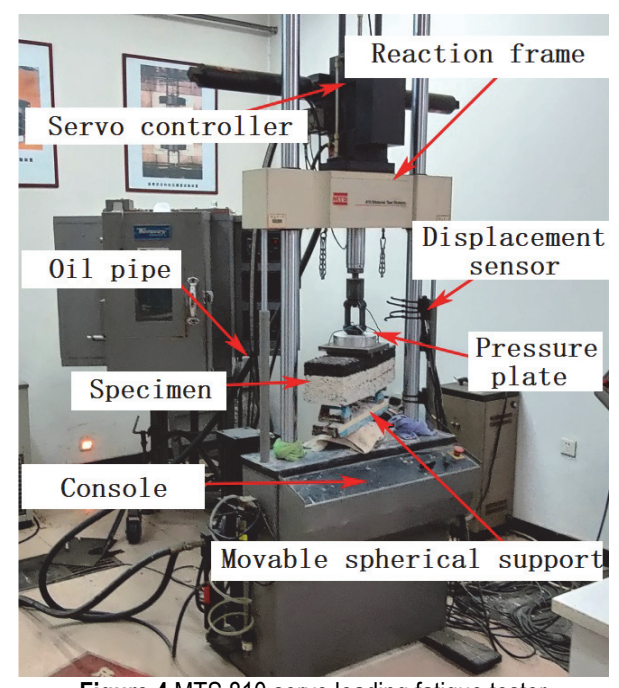

Figure 4 MTS 810 servo loading fatigue tester

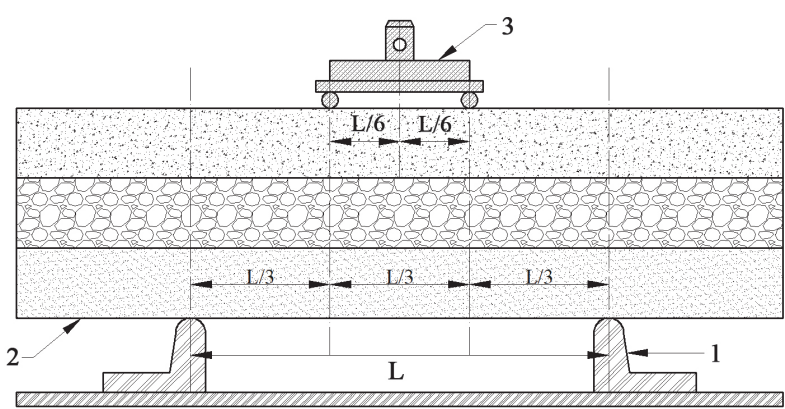

Figure 5 Loading scheme of specimens: (1) spherical movable support, (2) specimens, (3) upward pressure head

In the test, fatigue loads were applied through the Havesine fluctuating cyclic mode and the loading frequency was $10 \mathrm{~Hz}$. The loading waveform is shown in Fig. 6. Before loading, the flexural-tensile strength test of girders was performed first to determine the maximum bending strengths of reinforced and non-reinforced specimens and then the appropriate stress loading level was chosen. During fatigue loading, specimens were prepressed with 0.2 times of stress level to decrease the effects of bad contact. Subsequently, stress was increased to the design loads and the loading process continued until thorough compressive failure of specimens.

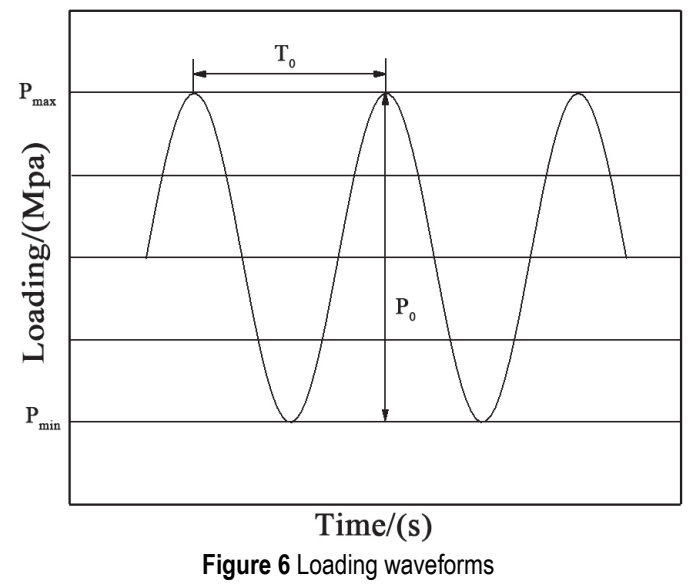

In Fig. 6, $P_{\max }$ is the maximum load and $P_{\min }$ is the minimum load. $P_{\min }=0.02 \times P_{\max }$ and $P_{0}=P_{\max }-P_{\min }, T_{0}$ is the loading cycle and $T_{0}=1 / f$, where $f$ is the loading frequency. The standard frequency is $10 \mathrm{~Hz}$.

\subsection{Data Acquisition and Measurement Contents}

In this test, the data acquisition system includes a deformation measurement instrument and a time recorder. A displacement acquisition system was adopted to measure the bending deformation of specimens to understand the load-deformation laws at bending deformation of specimens. The output loads of the hydraulic servo and the number of loading actions were collected and processed by a computer. According to test objectives, measurement contents of this test include the following:

(1) Maximum bending failure strength of specimens.

(2) Displacement of specimens at top loading points.

(3) The fatigue loading level of specimens and the number of loading actions under different stress levels. 


\section{RESULTS AND ANALYSIS}

\subsection{Analysis of Flexural-Tensile Strength Test Results 4.1.1 Group A}

Four flexural-tensile strength tests of non-reinforced composite macadam base specimens (Group A) were carried out. The displacement deformation yield curves in each test can be gained through a data acquisition system. Representative bending failure yielding curve can be recognized through error analysis and processing of data (Fig. 7).

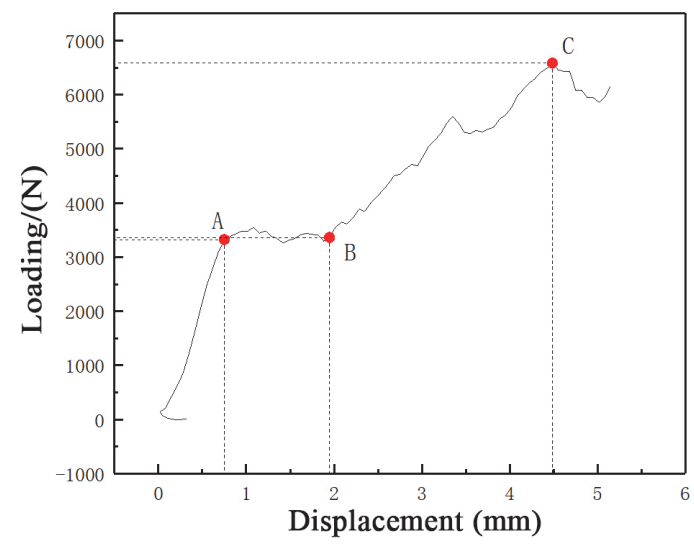

Figure 7 Bending failure curve of macadam foundation

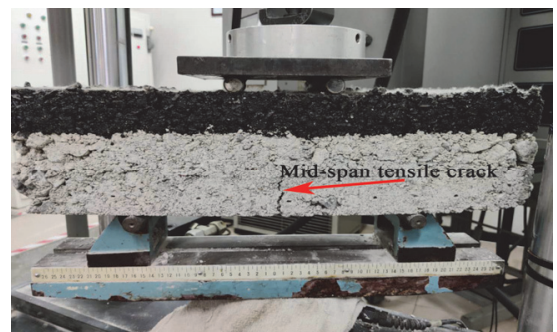

(a) Cement-stabilized macadam foundation generates a cut-through crack at point $A$

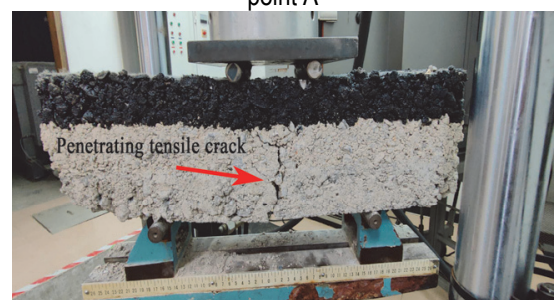

(b) Cracks propagates to bottom of the asphalt

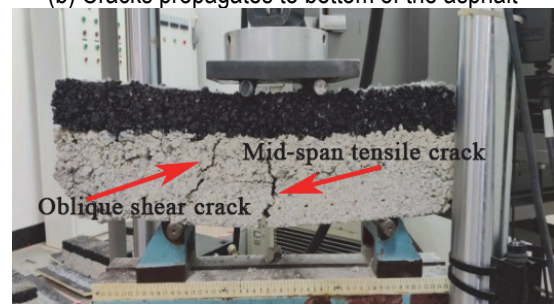

(c) Specimen generates an oblique shearing fracture near the suppose in

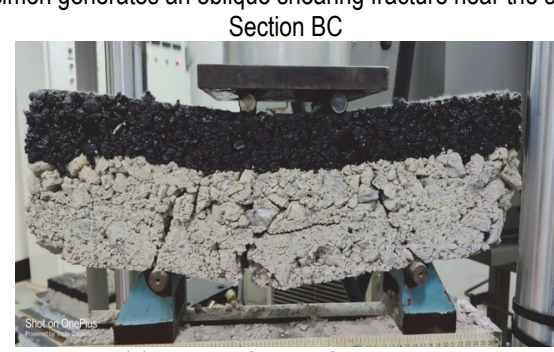

(d) Bending failure of specimens Figure 8 Bending failure of specimens
Fig. 7 shows that the curve is smooth and rises quickly at the beginning of loading. At this moment, the cementstabilized macadam base has good rigidity and high bearing capacity, resulting in the slow growth of overall displacement. When the curve reaches Point A, the cementstabilized macadam base develops cut-through reflection cracks. For specimens without geogrids, the cementstabilized macadam base cracks instantly under loads once it generates cut-through cracks. Then, the reflection cracks transmit to the bottom of the asphalt surface directly. In Section AB, the bearing capacity of cement-stabilized macadam base declines significantly and the macadam base particles move toward two sides under the loading effect. Displacement deformation of specimens grows quickly, and the width of reflection cracks is further expanded. After arriving at point $\mathrm{B}$, the macadam base aggregates are rearranged, and specimens reach a new dynamic balance. Section $\mathrm{BC}$ is the failure stage of the asphalt surface. At this stage, the cement-stabilized macadam base begins to develop oblique shearing fractures because the position near the support and the macadam layer avoids stress concentration through its loose structure and decreases flexural-tensile stress of the sub-base course of the bottom base. The asphalt surface develops bending deformations under loads and the displacement increases slowly until the breakage of the specimens. The flexuraltensile strength and displacement of specimens at Point $\mathrm{C}$ are $6.583 \mathrm{kN}$ and $4.48 \mathrm{~mm}$, respectively. Specific failure modes of specimens are shown in Fig. 8.

\subsubsection{Group B}

The bending failure curve of the reinforced macadam base (Group B) is acquired through the same test method of Group A. Fig. 9 shows that after geogrids are added in, the overall failure curves of specimens continued to rise at the same rate throughout the test, with a sharp increase stage of displacement in Fig. 7. The flexural-tensile strength at point $\mathrm{D}$ reaches $8.615 \mathrm{kN}$ and the displacement is $9.33 \mathrm{~mm}$. The flexural-tensile strength of reinforced macadam base is increased by $30.86 \%$ and the failure displacement is increased by $108.25 \%$ as compared to those of the macadam base. Moreover, the curve has a zigzag-shaped fluctuation near the failure point, thereby indicating that the macadam base aggregates are compacted further because of the lacing reinforcement of geogrids when the specimen will reach the failure point.

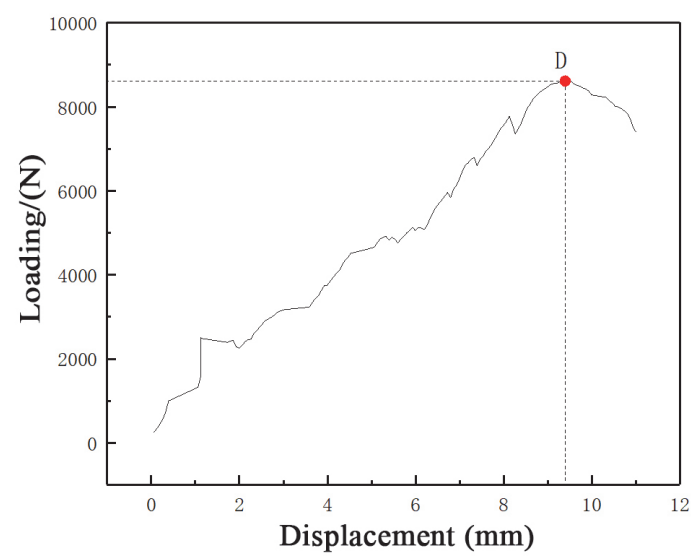

Figure 9 Bending failure curves of reinforced macadam foundation 
Therefore, the macadam base regains its bearing capacity and specimens can further generate bending deformation rather than breakage. The specimen develops overall failure only when the breakage of geogrids occurs. The specific failure mode is shown in Fig. 10.

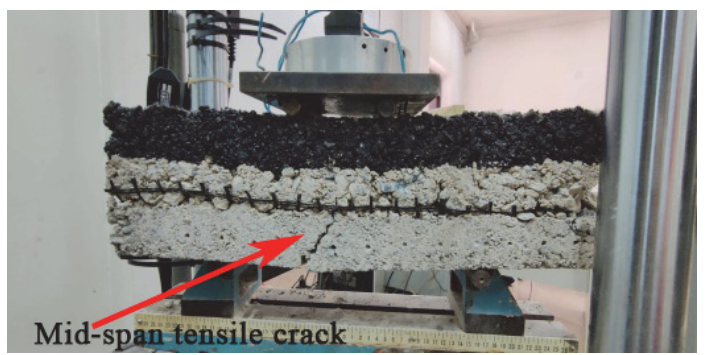

(a) Cement-stabilized macadam foundation of reinforced specimens

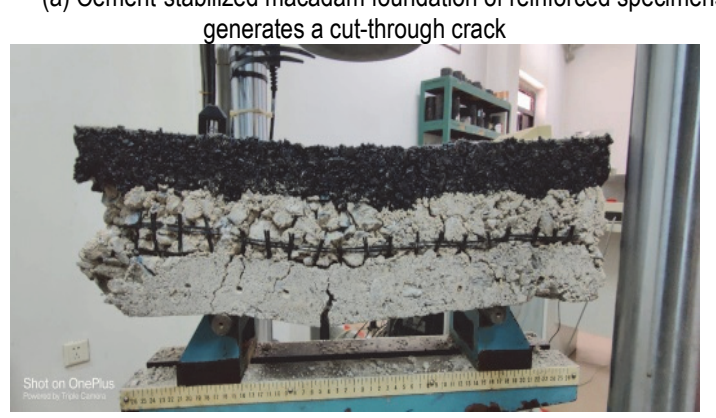

(b) Breakage failure of reinforced specimens

Figure 10 Bending failure modes of reinforced macadam foundation specimens
Fig. 10a shows that after geogrids are applied, the macadam base can isolate the cut-through crack from the cement-stabilized macadam base effectively even though the crack propagates. Hence, the crack will be difficult to transmit to the bottom of the asphalt surface along the macadam base directly. With the gradual increase of displacement, geogrids are laced further, giving the specimen a larger deformation capacity at failure. Fig. 10b shows that adding geogrids can effectively decrease the propagation of the oblique shearing fracture near the support at the moment of failure of the specimen as shown by the reduction of width and transmission speed of the fracture. Hence, the overall bending resistance of the pavement structure is enhanced to inhibit the transmission of reflection cracks.

\subsection{Analysis of Three-Point Bending Fatigue Test}

In this test, five groups of stress levels were chosen to Group A and Group B, respectively. Each stress level used five specimens for parallel tests. After the test, data error and the mean of the five specimens were analyzed to decreased discreteness. Any three groups of similar test data for each stress level were chosen as available data (Tab. 5). The numbers of fatigue loading cycles of Group A and Group B under different stress levels are shown in Fig. 11.

\begin{tabular}{|c|c|c|c|c|c|c|}
\hline \multirow{2}{*}{$\begin{array}{c}\text { Stress level } \\
0.6\end{array}$} & \multicolumn{3}{|c|}{ Group A } & \multicolumn{3}{|c|}{ Group B } \\
\hline & 126583 & 116631 & 130672 & 161239 & 141370 & 150126 \\
\hline 0.7 & 95946 & 90920 & 92463 & 114240 & 105239 & 124652 \\
\hline 0.76 & 24987 & 28783 & 20546 & 67702 & 76333 & 62835 \\
\hline 0.78 & 11881 & 15006 & 13620 & 49115 & 57222 & 37406 \\
\hline 0.8 & 4889 & 6999 & 4138 & 25648 & 22348 & 18964 \\
\hline
\end{tabular}

Tab. 5 and Fig. 11 show that although the number of fatigue loading cycles of the reinforced specimens is higher than the non-reinforced specimens under the stress level of 0.6 or 0.7 , the difference is relatively small because the initial deformation of specimens is small under low-stress levels and the geogrids are not stretched fully to develop the best reinforcement effect. The fatigue life of the reinforced specimens is $21.09 \%$ higher on average compared to that of non-reinforced specimens. When the stress level is increased to 0.76 , the fatigue life of the nonreinforced specimens is only $1 / 4$ of that under 0.7 stress level, while the fatigue life of reinforced specimens can reach $60 \%$ of that under 0.7 stress level, thereby indicating the poor dynamic stability of pavement structure with a macadam base under high-stress levels. When the cementstabilized macadam base generates a cut-through crack and the bearing capacity declines considerably, the loose macadam base has difficulty bearing the long-term dynamic vehicle loads from the pavement and it is extremely easy for the pavement structure to develop bending fatigue failures. After geogrids are added, their interlocking effect on the macadam base can strengthen the structural performance of the pavement, improve the stability of the macadam base under dynamic loads, and increase the bending fatigue life of pavement structure significantly. Hence, reinforcement can improve bending fatigue properties of pavement structure with macadam base significantly.

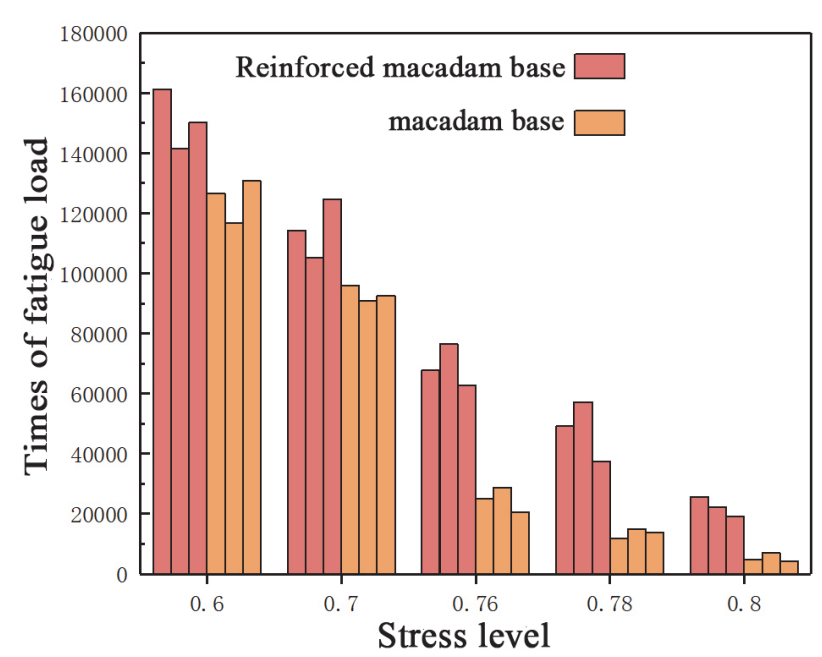

Figure 11 Number of fatigue loading cycles under different stress levels

Fig. 11 shows that with the continuous increase of stress level to 0.8 , the macadam base develops low-cycle fatigue failure, while the reinforced macadam base structures can still maintain a high post-fatigue life of about 20,000 cycles. No strict boundary exists between highcycle fatigue and low-cycle fatigue. However, it is a common practice in engineering to classify fatigue problems with failure cycles $N_{f}<10^{4}$ into the range of lowcycle fatigue. When low-cycle fatigue failure occurs, local stresses on the specimen have exceeded the yield limit of 
materials and the specimen will form a relatively large plastic zone as a response to the alternating stress. Macrocracks are very easy to be developed in this plastic zone. Hence, low-cycle fatigue failures of pavement structure should be strictly prevented to obtain more reliable and durable pavement structures. Geogrid reinforcement can help pavement structure with macadam base to maintain high-cycle fatigue failures under 0.8 stress level. It relieves brittle failure of the pavement structure and prolongs its fatigue life effectively.

\subsection{Crack Propagation and Comparative Test Results}

In the comparative test of crack propagation between reinforced and non-reinforced specimens, the stress level was 0.7 and fatigue cracks under specific numbers of loading cycles were recorded. Details are shown in Fig. 12 and Fig. 13. Based on a comparison of crack propagation in Fig. 12 and Fig. 13 under different numbers of loading cycles, the reflection crack of Group A has cut through the whole base structure at 30,000 loading cycles, and a clear oblique shearing fracture developed at edges of the macadam base and near supports at 70,000 loading cycles. Moreover, Group A broke at 90,000 loading cycles.
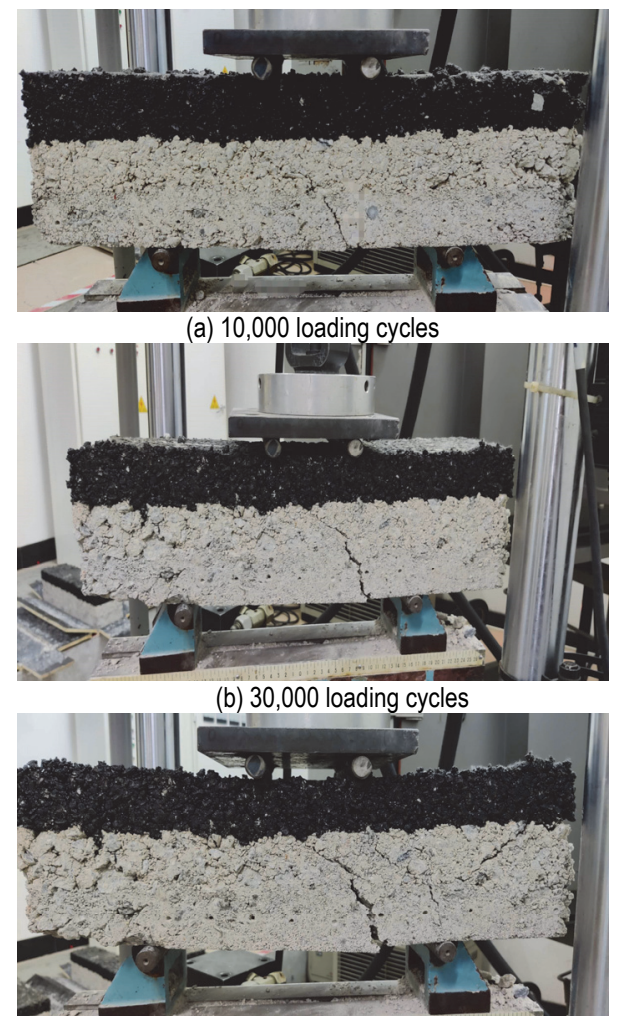

(c) 70,000 loading cycles

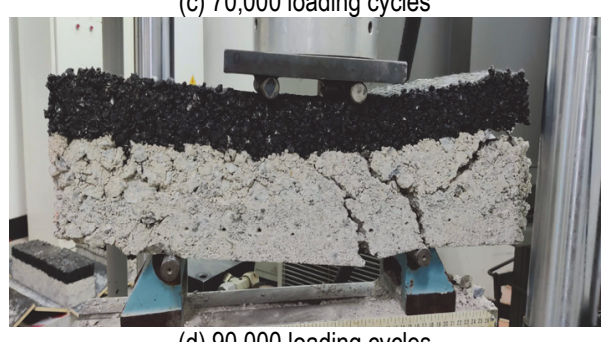

(d) 90,000 loading cycles

Figure 12 Number of fatigue loading cycles under different stress levels

A serious bending deformation at the top asphalt surface can be observed and the asphalt surface separated from the macadam layer. The specimen is broken seriously. On the contrary, Group B has no reflection crack that runs through the whole base structure at 70,000 loading cycles and only developed reflection crack that runs through the cement-stabilized macadam base at the midspan. This reflection crack did not propagate upward along the original cracking surface after it cut through the cementstabilized macadam base because of the lacing reinforcement of geogrids. The shear fracture near the support of Group B is far smaller than that of Group A. Even when the loading cycles reached 90,000, the reflection crack at the midspan of Group B still did not propagate to the macadam base and the base structure maintained good integrity. In the whole test, the bending deformation of Group B was kept within a small range, while the deformation of Group A fluctuated significantly after 30,000 loading cycles, and the specimen was broken obviously. To sum up, reinforcement can inhibit the transmission of reflection crack on the base course, decrease cracking of the macadam base effectively, and delay the development of oblique shearing fracture near the support.

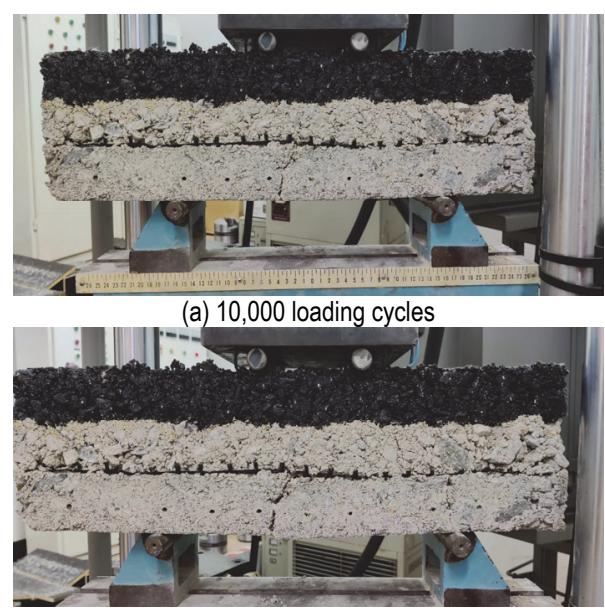

(b) 30,000 loading cycles

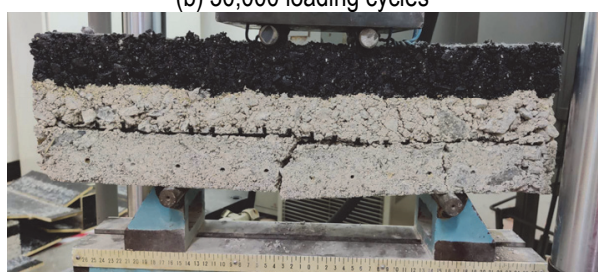

(c) 70,000 loading cycles

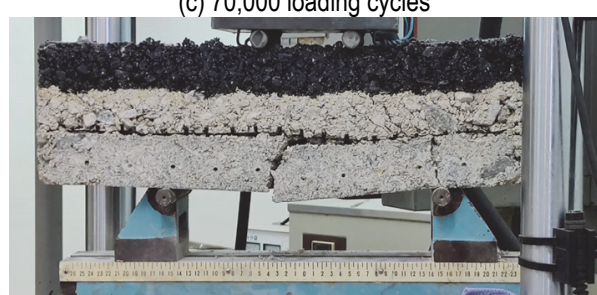

(d) 90,000 loading cycles

Figure 13 Fatigue crack propagation on the reinforced macadam foundation structure

\subsection{Yield Curves under Different Stress Levels}

Fatigue failure curves of Group A and Group B under different stress levels were plotted in a unified coordination system (Fig. 14). 
Fig. 14a shows that the displacement of Group A at the failure point under a low-stress level is between $4 \mathrm{~mm}-8$ $\mathrm{mm}$. The graded macadam base cannot bear tensile stress and thus, it cracked and was cut through immediately upon cracking failure of the cement-stabilized macadam base. Under this circumstance, the asphalt surface bears the loads alone giving rise to the possibility of developing fatigue cracks after reaching a certain displacement. Therefore, the specimen loses its bearing capacity completely and breaks quickly. When the stress level is increased to 0.76 or higher the fatigue life attenuates significantly, and the curve is shortened quickly. Moreover, the average bending displacement at failure is $4 \mathrm{~mm}-6 \mathrm{~mm}$. To sum up, the ultimate stress level of the pavement structure with the macadam base is between $0.7-0.76$. At this stress level interval, the dynamic stability of the structure declines significantly and the structure can easily develop brittle breakage under fatigue loads. The cement-stabilized macadam base develops low-cycle brittle failures due to high initial loads and is cracked completely before dispersion and attenuation of surface loads, thereby losing its function as a flexible base.

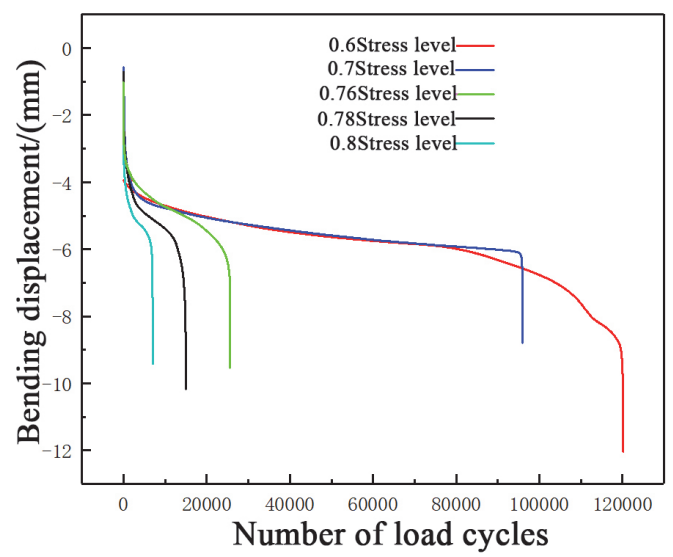

(a) Failure curve of Group A under different stress levels

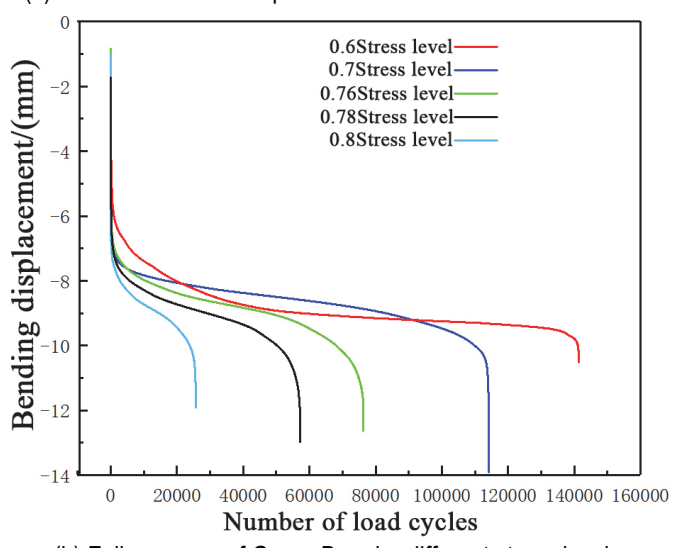

(b) Failure curve of Group B under different stress levels

Figure 14 Failure curves under different stress levels

Fig. 14b shows that the displacement of reinforced specimens at the failure point under low-stress levels is between $8-10 \mathrm{~mm}$, which is about $50 \%$ higher than nonreinforced specimens. For reinforced specimens, geogrids are laced when the specimen develops bending deformation continuously and the tight geogrids squeeze the macadam aggregates. Loads on the asphalt surface are dispersed and attenuated by macadam base first. Subsequently, the residual bending stresses are transformed partially into the frictional resistance of the geogrid-soil interface. Finally, the compressive stress transmitted to the cement-stabilized macadam base is decreased significantly, thereby enabling the cementstabilized macadam base to maintain a relatively long fatigue life after the formation of a cut-through crack. The failure curve is not attenuated significantly when the stress level is higher than 0.7. The reinforced specimens still can maintain relatively large failure displacement when the stress level is 0.76 or 0.78 . The macadam base can develop its stress-buffering effect to the maximum extent. As a result, the fatigue life of the base structures for the pavement under high dynamic vehicle loads is prolonged and the durability of the base structure increased.

\section{CONCLUSIONS}

This study proposes a reinforced composite macadam base structure to eliminate the reflection cracks on the traditional semi-rigid foundation structure. In the proposed structure, geogrids are applied in the space between macadam base and cement-stabilized macadam base to improve the dynamic stability of the whole structure and further strengthen the bending resistance and fatigue life of the pavement structure. A three-point bending fatigue test and a flexural-tensile strength test have been implemented on reinforced and non-reinforced specimens. Five different stress levels have been set in the cyclic loading test. Failure modes and fatigue failure curves of specimens under different stress levels have been analyzed. Based on the comparison of the test data of reinforced and nonreinforced specimens, it proves that geogrid reinforcement can improve the bending fatigue properties of macadam base structures. Some major conclusions can be drawn:

(1) Transmission of reflection cracks on the sub-base course cannot be prevented effectively by the macadam base structure alone. When the crack cuts through the subbase course, it will propagate to the bottom of the asphalt surface directly along the macadam base. Adding geogrids can prevent the transmission of reflection cracks at midspan of the sub-base course and delay generation of the oblique shearing fracture near the support.

(2) Based on the analysis of flexural-tensile strength test results of reinforced and non-reinforced specimens, the macadam base structures easily develop brittle failures under bending stresses and the bending displacement at failure is relatively small. After geogrids are applied, the bending strength and displacement at the failure of specimens are increased by $30.86 \%$ and $108.25 \%$, respectively. These results reflected that reinforcement can increase ultimate displacement deformation at structural failure and strengthen the resistance of pavement structures with a macadam base to bending deformation.

(3) Analysis of the number of loading cycles under different stress levels indicates that the fatigue life of macadam base structures is shortened significantly when the stress level is higher than 0.7 , indicating that the ultimate stress level of macadam base structures is between $0.7-0.76$. After geogrids are applied, the bending fatigue life of pavement structure is prolonged significantly under different stress levels. Moreover, the geogrid reinforcement prevents sharp attenuations of fatigue life as approaching to the ultimate stress level, avoids low-cycle 
fatigue failure of base structures under high-stress levels, and increases dynamic stability of macadam base structures under cyclic loading conditions effectively.

The proposed reinforcement macadam base structure is characterized by its simple and high-efficiency construction and low engineering cost. It has been proved to have good mechanical properties and dynamic stabilities through laboratory bending strength tests and bending fatigue tests. A reinforced macadam base can be used as a new reinforcement structure of macadam base and should be promoted at large-scale in practical engineering. However, this study is limited by mechanical performance indices because of the small number of specimens. Thus, further research on the influences of other material parameters in the reinforced macadam base structure is still needed.

\section{REFERENCES}

[1] Zhou, G., Li, R. K., Wang, H. M., \& Li, W. H. (2016). Test on Performance of Reflective Cracking Resistance of Warp Knitting Fiberglass-polyester Paving Mat. China Journal of Highway and Transport, 29(2), 16-22.

[2] Tomás-Espín, A. \& Sanchez-Olivares, G. (2018). Optimum reinforcement and sizing of concrete cross sections subjected to flexural and axial loading. DYNA, 93(5). 512-520. https://doi.org/10.6036/8676

[3] Xin, Q., Dou, Y., Zhang, J., \& Li, S. (2018). Deflection calculation of partially prestressed concrete beams under heavy duty. DYNA, 94(3), 301-307. https://doi.org/10.6036/8737

[4] Zhou, L. J., Zheng, M. L., Ma, Q., \& Ma, J. G. (2016). Simulation of reflection crack propagation path for complex pavement with stress absorbing layer. Journal of Railway Science and Engineering, 13(8), 1507-1514.

[5] Harikumar, M., Sankar, N., \& Chandrakaran, S. (2020). Effect of Multi-Directional Reinforcements on the Settlement and Heave Characteristics of Foundations on Sand. Geotechnical and Geological Engineering, 1-11. https://doi.org/10.1007/s10706-020-01506-z

[6] Ouria, A., Mahmoudi, A., \& Sadeghpour, H. (2020). Effect of the Geotextile Arrangement on the Bearing Capacity of a Strip Footing. International Journal of Geosynthetics and Ground Engineering, 6(36), 1-14. https://doi.org/10.1007/s40891-020-00219-w

[7] Ban, H., Im, S., Kim, Y. R., \& Jung, J. S. (2018). Laboratory tests and finite element simulations to model thermally induced reflective cracking of composite pavements. International Journal of Pavement Engineering, 19(3), 220230. https://doi.org/10.1080/10298436.2017.1279491

[8] Lu, L., Zhang, J. J., Ma, S. W., Wang, Z. J., \& Chen, Z. X. (2020). Dynamic response of prestressed wrap-reinforced earth retaining walls. Chinese Journal of Geotechnical Engineering, 42(2), 344-353.

[9] Liu, M. C., Zho, X. H., \& Ge, Z. S. (2014). Design and Computational Method for Bridge Approach Reinforced Backfills. China Journal of Highway and Transport, 27(2), 17-26.

[10] Hashemolhosseini, H. \& Mirmohamadsadeghi, M. (2020). Investigating the Effect of Geocell Changes on Slope Stability in Unsaturated Soil. Technical Gazette, 14(1), 6675. https://doi.org/10.31803/tg-20190606115822

[11] Zhao, M. H., Zhen, D. X., Liu, M., \& Ou, Q. (2020). Deformation analysis of geocell-reinforced body under embankment load considering soil arch effect. Chinese Journal of Geotechnical Engineering, 42(4), 601-609.

[12] Zhou, D., Ma, Z. X., Liu, L. P., \& Sun, L. J. (2020). Study on Fatigue Performance of In-service Asphalt Pavement Based on Full-scale Accelerated Loading Test. Journal of Highway and Transportation Research and Development, 37(1), 1724.

[13] Zhen, S. X., Zhang, X. N., Meng, S. T., \& Ren, W. X. (2012). Fatigue Damage in Asphalt Layer Based on ALF Accelerated Loading Test. Journal of Highway and Transportation Research and Development, 29(1), 18-22.

[14] Ji, X., Zheng, N., Hou, Y., \& Niu, S. (2013). Application of asphalt mixture shear strength to evaluate pavement rutting with accelerated loading facility (ALF). Construction and Building Materials, 41, 1-8. https://doi.org/10.1016/j.conbuildmat.2012.11.111

[15] Li, Y., Song, G., \& Cai, J. (2017). Mechanical response analysis of airport flexible pavement above underground infrastructure under moving wheel load. Geotechnical and Geological Engineering, 35(5), 2269-2275. https://doi.org/10.1007/s10706-017-0242-8

[16]Valli, S. A. \& Kolathayar, S. (2019). Effect of Natural Rubber, Carbon Black, and Copper Slag as Construction Materials in Flexible Pavement: A Review. In: Pulugurtha, S., Ghosh, I., Biswas, S. (Eds). Advances in Transportation Engineering, 59-64. https://doi.org/10.1007/978-981-13-7162-2_6

[17] Öcal, A. \& Pekcan, O. (2021). Cuckoo Search Based Backcalculation Algorithm for Estimating Layer Properties of Full-Depth Flexible Pavements. Dey, N. (Ed.). Applications of Cuckoo Search Algorithm and its Variants, 229-252. https://doi.org/10.1007/978-981-15-5163-5_10

[18] You, L., Man, J., Yan, K., Wang, D., \& Li, H. (2020). Combined Fourier-wavelet transforms for studying dynamic response of anisotropic multi-layered flexible pavement with linear-gradual interlayers. Applied Mathematical Modelling, 81, 559-581. https://doi.org/10.1016/j.apm.2020.01.031

[19] Patil, V., Joshi, P., Kale, S., \& Pingle, T. (2019). Design of Flexible Pavement on Black Cotton Soil Stabilized with RBI Grade 81. In: Agnihotri, A., Reddy, K., Bansal, A. (Eds), Environmental Geotechnology, 193-200. https://doi.org/10.1007/978-981-13-7010-6_18

[20]Sayyed, S. S., Patil, R. P., Tapase, A., Attar, A. C., \& Chandak, P. G. (2018). Review and assessment of flexible pavement. In: Chen, D., Kim, S., Tapase, A. (Eds), Civil Infrastructures Confronting Severe Weathers and Climate Changes Conference, 139-149. https://doi.org/10.1007/978-3-319-96241-2_12

[21] Habbouche, J., Hajj, E. Y., Morian, N. E., Sebaaly, P. E., \& Piratheepan, M. (2017). Reflective cracking relief interlayer for asphalt pavement rehabilitation: from development to demonstration. Road Materials and Pavement Design, 18(4), 30-57. https://doi.org/10.1080/14680629.2017.1389080

[22] Peng, B., Tong, X. Y., Cao, N., Fan, X. Y., \& Tian, Y. H. (2018). Research on anti-cracking and road performance of lean asphalt gravel intermediate structural Layer. Journal of Dalian University of Technology, 54(4), 385-391.

[23] Pan, R. \& Cheng, P. (2015). Grading design and road use performance of warm mix rubber asphalt mixture suitable for stress absorbing layer in cold region. World Information on Earthquake Engineering, 31(1), 161-166.

[24] Gonzalez-Torre, I., Calzada-Perez, M. A., Vega-Zamanillo, A., \& Castro-Fresno, D. (2015). Experimental study of the behaviour of different geosynthetics as anti-reflective cracking systems using a combined-load fatigue test. Geotextiles and Geomembranes, 43(4), 345-350. https://doi.org/10.1016/j.geotexmem.2015.04.001

[25] Saride, S. \& Kumar, V. V. (2017). Influence of geosyntheticinterlayers on the performance of asphalt overlays on precracked pavements. Geotextiles and Geomembranes, 45(3), 184-196. https://doi.org/10.1016/j.geotexmem.2017.01.010

[26] Correia, N. S. \& Zornberg, J. G. (2016). Mechanical response of flexible pavements enhanced with geogridreinforced asphalt overlays. Geosynthetics International, 23(3), 183-193. https://doi.org/10.1680/jgein.15.00041 
[27] Hadi, M. N. \& Al-Hedad, A. S. (2020). Flexural fatigue behaviour of geogrid reinforced concrete pavements. Construction and Building Materials, 249, 1-10. https://doi.org/10.1016/j.conbuildmat.2020.118762

[28] Saha, D. C. \& Mandal, J. N. (2020). Potential use of reinforced reclaimed asphalt pavement (RAP) and wet mix macadam (WMM) mixture in flexible pavement construction. International Journal of Pavement Research and Technology, 13(4), 352-360. https://doi.org/10.1007/s42947-020-0080-6

[29] Sun, X., Han, J., Fei, J., Guo, J., \& Zhang, W. (2020). Geosynthetic-stabilized flexible pavements: Solution derivation and mechanistic-empirical analysis. Geotextiles and Geomembranes, 48(4), 468-478.

https://doi.org/10.1016/j.geotexmem.2020.02.005

\section{Contact information:}

Hongjun JING, PhD, Professor

(Corresponding author)

1) School of Architectural and Civil Engineering, $X^{i}$ an University of Science and Technology, Shaanxi Province, China,

2) Road Engineering Research Center of Xi' an University of Science and Technology, Shaanxi Province, China,

Room 2009, No.58 Yanta Road, Shaanxi Province, China

E-mail: jinghonhjun@xust.edu.cn

\section{Mingjie GOU, MS}

1) School of Architectural and Civil Engineering, Xi' an University of Science and Technology, Shaanxi Province, China,

2) Road Engineering Research Center of $\mathrm{Xi}^{\prime}$ an University of Science and Technology, Shaanxi Province, China,

Room 2009, No.58 Yanta Road, Shaanxi Province, China

E-mail: 787267175@qq.com

\section{Lichen SONG, MS}

1) School of Architectural and Civil Engineering, $X i$ ' an University of Science and Technology, Shaanxi Province, China,

2) Road Engineering Research Center of $X_{i}^{\prime}$ an University of Science and Technology, Shaanxi Province, China,

Room 2009, No.58 Yanta Road, Shaanxi Province, China

E-mail: 1249138957@qq.com 